PROCEEDINGS OF THE

AMERICAN MATHEMATICAL SOCIETY

Volume 131, Number 4, Pages 1233-1241

S 0002-9939(02)06646-7

Article electronically published on September 17, 2002

\title{
UNIVALENT MAPPINGS AND INVARIANT SUBSPACES OF THE BERGMAN AND HARDY SPACES
}

\author{
BRENT J. CARSWELL
}

(Communicated by Juha M. Heinonen)

\begin{abstract}
In both the Bergman space $A^{2}$ and the Hardy space $H^{2}$, the problem of determining which bounded univalent mappings of the unit disk have the wandering property is addressed. Generally, a function $g$ in $H^{\infty}$ has the wandering property in $X$, where $X$ denotes either $A^{2}$ or $H^{2}$, provided that every $g$-invariant subspace $M$ of $X$ is generated by the orthocomplement of $g M$ within $M$. It is known that essentially every function which has the wandering property in either space is the composition of a univalent mapping with a classical inner function, and that the identity mapping has this property in both spaces. Consequently, weak-star generators of $H^{\infty}$ also have the wandering property in both settings. The present paper gives a partial converse to this, and shows that in both settings there is a large class of bounded univalent mappings which fail to have the wandering property.
\end{abstract}

\section{INTRODUCTION}

The Bergman space $A^{2}$ consists of all functions $f$ analytic in the unit disk $\mathbb{D}=$ $\{z \in \mathbb{C}:|z|<1\}$ for which the norm

$$
\|f\|=\left(\int_{\mathbb{D}}|f|^{2} d \sigma\right)^{1 / 2}
$$

is finite. Here $d \sigma$ denotes normalized Lebesgue area measure in $\mathbb{D}$. The space $A^{2}$ is a Hilbert space, equipped with the inner product

$$
\langle f, g\rangle=\int_{\mathbb{D}} f \bar{g} d \sigma .
$$

A theorem of Aleman, Richter, and Sundberg [4 asserts that every closed $z$ invariant subspace $M$ of $A^{2}$ is generated by the orthocomplement of $z M$ within $M$. Similarly, a well-known result of Beurling [5] shows that this property also holds in the Hardy space $H^{2}$. Motivated by results of [7] and [12], the present paper investigates the extent to which this property extends to $\varphi$-invariant subspaces of both $A^{2}$ and $H^{2}$, where $\varphi$ is some given bounded univalent mapping of the unit disk. Specifically, in both the Bergman and Hardy spaces, we consider the problem of determining which bounded univalent mappings $\varphi$ have the property that every $\varphi$-invariant subspace $M$ is generated by the orthocomplement of $\varphi M$ within $M$.

Received by the editors May 22, 2001 and, in revised form, November 30, 2001.

2000 Mathematics Subject Classification. Primary 30H05, 46E20, 46E22.

Key words and phrases. Bergman space, Hardy space, wandering property, invariant subspace, multiplication operator, reproducing kernel, weak-star generator. 
For a given function $g$ in $H^{\infty}$, a closed subspace $M$ of $A^{2}$ is said to be $g$-invariant provided $g M \subset M$, that is, if $g f \in M$ whenever $f \in M$. For example, if $f \in A^{2}$ and $\left\{a_{n}\right\}$ are the zeros of $f$ (repeated according to multiplicity), then the subspace $M$ consisting of all functions in $A^{2}$ which vanish to at least the prescribed order on the sequence $\left\{a_{n}\right\}$ is a $g$-invariant subspace, for every $g$ in $H^{\infty}$. An invariant subspace $M$ of this type is commonly called a zero-based subspace.

For a subset $E \subset A^{2}$, let $[E]_{g}$ denote the smallest closed $g$-invariant subspace of $A^{2}$ which contains $E$. For example, the singly-generated $g$-invariant subspace $[f]_{g}$ is simply the $A^{2}$-closure of the functions of the form $(P \circ g) f$ for which $P$ is a polynomial. (It is common to abuse notation and write $[f]_{g}$ instead of $[\{f\}]_{g}$.)

It is useful to point out that all $z$-invariant subspaces are $g$-invariant for each $g$ in $H^{\infty}$. (This is easy to see by first proving it for $g(r z)$ and letting $r$ approach 1.) However, the converse is false. For example, if $g(z)=z^{2}$ and $M$ consists of the even functions in $A^{2}$, then $M$ is easily seen to be $z^{2}$-invariant, but not $z$-invariant.

A function $g$ in $H^{\infty}$ is said to have the wandering property in $A^{2}$ provided that

$$
M=[M \ominus g M]_{g}
$$

for every $g$-invariant subspace $M$ of $A^{2}$. Here $M \ominus g M$ denotes the orthocomplement of $g M$ within $M$. (We similarly define what it means for a function to have the wandering property in $H^{2}$ by replacing $A^{2}$ in the above definitions with $H^{2}$.) The result of Aleman, Richter, and Sundberg [4] can therefore be phrased by saying that the identity mapping $g(z)=z$ has the wandering property in $A^{2}$. In $H^{2}$, the identity map has the wandering property by Beurling's theorem [5].

It was shown in [7] that essentially every function $g$ in $H^{\infty}$ which has the wandering property in $A^{2}$ must be of the form $g=\varphi \circ h$ for some Riemann map $\varphi$ and some inner function $h$. A similar result for the Hardy space was proved in 12. Furthermore, it was shown in [7] that there are inner functions without the wandering property in $A^{2}$. This contrasts with the Hardy space situation, where the Wold decomposition theorem implies that when $g$ is inner, every $g$-invariant subspace $M$ of $H^{2}$ has the structure $M=[M \ominus g M]_{g}$.

Based on the main results of [7] and [12], it is natural to ask which bounded univalent mappings have the wandering property in either $A^{2}$ or $H^{2}$. In addition to what was mentioned previously, it was proved in [12] that if $g$ has the form $g=\varphi \circ h$, where $h$ is an inner function and $\varphi$ is a weak-star generator of $H^{\infty}$, then $g$ has the wandering property in $H^{2}$. In particular, weak-star generators of $H^{\infty}$ have the wandering property in $H^{2}$. In the present paper, we observe that weak-star generators of $H^{\infty}$ also have the wandering property in $A^{2}$, and we give a partial converse to this fact in both the Bergman and Hardy space settings. This partial converse can be given in terms of a relationship between the reproducing kernels corresponding to certain pullback measures. Furthermore, appealing to the work of [2] and [3], we will show that in both settings there is a large class of bounded univalent mappings which fail to have the wandering property.

\section{MAin Results}

Given $g$ in $H^{\infty}$, we use the notation $\operatorname{Lat}_{A^{2}}(g)$ to denote the lattice of $g$-invariant subspaces of $A^{2}$. Our first main result is the following theorem.

Theorem 2.1. Let $\varphi$ be a bounded univalent mapping of $\mathbb{D}$, with $\varphi(0)=0$. Then $\operatorname{Lat}_{A^{2}}(\varphi)=\operatorname{Lat}_{A^{2}}(z)$ if and only if 
(i) $M=[M \ominus \varphi M]_{\varphi}$ for every $M$ in $\operatorname{Lat}_{A^{2}}(\varphi)$, and

(ii) $[f]_{\varphi} \ominus \varphi[f]_{\varphi}=[f]_{z} \ominus z[f]_{z}$ for every $f$ in $A^{2}$.

Since the condition $\operatorname{Lat}_{A^{2}}(\varphi)=\operatorname{Lat}_{A^{2}}(z)$ is equivalent to the condition that $\varphi$ is a weak-star generator of $H^{\infty}$ (see Lemma 3.1 below), we observe that Theorem 2.1] says that weak-star generators of $H^{\infty}$ have the wandering property in $A^{2}$. Furthermore, Theorem 2.1 gives a partial converse of this fact.

In addition, we will give a Hardy space version of Theorem 2.1, which appears later as Theorem 5.1 Moreover, we will obtain the next result.

Theorem 2.2. Suppose that $g \in H^{\infty}$ has the wandering property in $A^{2}$, and that $g(0)=0$. Then the following are equivalent:

(A) $g$ is univalent,

(B) every singly-generated $z$-invariant subspace of $A^{2}$ is of the form $[f]_{g}$ for some function $f$ in $A^{2}$,

(C) there exists a singly-generated $z$-invariant subspace of $A^{2}$ that is of the form $[f]_{g}$ for some $f$ in $A^{2}$.

We remark here that Theorem 2.2 implies, for instance, that if $g \in H^{\infty}$ with $g(0)=0$, and if for some zero-based subspace $M$ there is a function $f$ for which $M=[f]_{g}$, then either $g$ is univalent or $g$ fails to have the wandering property in $A^{2}$.

Finally, we will observe that if a bounded univalent mapping $\varphi$ has the wandering property in $A^{2}$ (respectively, $H^{2}$ ), then polynomials in $\varphi$ are dense in $A^{2}$ (respectively, $H^{2}$ ), and thus it will follow that in both the Bergman and Hardy space settings there is a large class of bounded univalent mappings which fail to have the wandering property. For emphasis, we state this as a corollary.

Corollary 2.3. Let $X$ denote either $A^{2}$ or $H^{2}$. There exist bounded univalent mappings $\varphi$ of the unit disk, with $\varphi(0)=0$, which fail to have the wandering property in $X$.

This paper is organized as follows. In Section 3, we give some useful facts about weak-star generators of $H^{\infty}$. In addition, we outline some well-known results about $z$-invariant subspaces of $A^{2}$ which will be used in the proof of both main results. In Section 4, we prove our results in the Bergman space setting, while in Section [5] we observe that the results in Section 4 have Hardy space analogs. In Section 6 we discuss a few examples that justify Corollary 2.3.

\section{Preliminaries}

Weak-star generators of $H^{\infty}$. We begin by collecting some facts about weakstar generators of $H^{\infty}$. One good source for this is Sarason's paper [14].

The weak-star topology of $H^{\infty}$ can be viewed as follows. Given $g$ in $H^{\infty}$ and $w$ in the unit circle $\mathbb{T}=\partial \mathbb{D}$, let $g^{*}(w)$ denote the radial limit of $g$ at $w$. By Fatou's Theorem, $g^{*}$ is defined almost everywhere in $\mathbb{T}$. The map $g \rightarrow g^{*}$ is an isometric isomorphism of $H^{\infty}$ onto a subspace of $L^{\infty}(\mathbb{T})$. This subspace is denoted $H^{\infty}(\mathbb{T})$. Since $L^{\infty}(\mathbb{T})$ is the dual of $L^{1}(\mathbb{T})$, it has a weak-star topology. Thus $H^{\infty}(\mathbb{T})$ inherits this topology, and the correspondence between $H^{\infty}$ and $H^{\infty}(\mathbb{T})$ induces the weakstar topology on $H^{\infty}$.

A function $\varphi$ in $H^{\infty}$ is called a weak-star generator of $H^{\infty}$ if polynomials in $\varphi$ are dense in the weak-star topology in $H^{\infty}$. It is well known that weak-star generators 
are necessarily univalent. If it happens that every function $f \in H^{\infty}$ is the weak-star limit of a sequence of polynomials in $\varphi$, then $\varphi$ is said to be a sequential weak-star generator of $H^{\infty}$. It is obvious that sequential generators are generators. However, not every generator is sequential [14].

In the same paper, Sarason showed that weak-star generators $\varphi$ can be characterized by the domains $\Omega=\varphi(\mathbb{D})$. For example, if $\Omega$ is a Jordan domain, then any mapping $\varphi$ which takes $\mathbb{D}$ onto $\Omega$ is a weak-star generator of $H^{\infty}$. The present paper is primarily concerned with univalent mappings $\varphi$ which are not weak-star generators. One of the simplest examples of a simply connected domain $\Omega$ for which the mapping $\varphi$ of $\mathbb{D}$ onto $\Omega$ is not a weak-star generator of $H^{\infty}$ is the slitted disk $\Omega=\mathbb{D} \backslash\left[\frac{1}{2}, 1\right)$. An example of a simply connected domain $\Omega$ without slits for which the map $\varphi$ taking $\mathbb{D}$ onto $\Omega$ is not a generator is a crescent. A crescent is a domain whose boundary consists of two Jordan curves which meet at exactly one point.

The proof of the following lemma, which links the property of being a weak-star generator of $H^{\infty}$ with the lattice of invariant subspaces, can be found in [6].

Lemma 3.1. A bounded univalent mapping $\varphi$ is a weak-star generator of $H^{\infty}$ if and only if $\operatorname{Lat}_{A^{2}}(z)=\operatorname{Lat}_{A^{2}}(\varphi)$.

The Hardy space version of Lemma 3.1 was proved in [13]:

Lemma 3.2. A bounded univalent mapping $\varphi$ is a weak-star generator of $H^{\infty}$ if and only if $\operatorname{Lat}_{H^{2}}(z)=\operatorname{Lat}_{H^{2}}(\varphi)$.

We remark that for every $g$ in $H^{\infty}$, we easily have $\operatorname{Lat}_{A^{2}}(z) \subseteq \operatorname{Lat}_{A^{2}}(g)$, and $\operatorname{Lat}_{H^{2}}(z) \subseteq \operatorname{Lat}_{H^{2}}(g)$.

Properties of $z$-invariant subspaces of the Bergman space. We now record some well-known facts about $z$-invariant subspaces of $A^{2}$ which we will use in the proofs of Theorems 2.1 and 2.2 The reader is directed to the books [8] and [11], or to the fundamental papers [4] and [10], for further details.

If $M=[h]_{z}$ for some function $h \in A^{2}$, then $M \ominus z M$ is one-dimensional and is spanned by the unique function $G$, called the extremal function for $M$, which maximizes $\operatorname{Re} f(0)$ over all functions $f$ in $M$ with $\|f\| \leq 1$. (Here, we assume that $h(0) \neq 0$, otherwise if $h$ has a zero of degree $n$ at zero, then we maximize $\operatorname{Re} f^{(n)}(0)$.) Furthermore, $G$ has the property that for every $f$ in $M$,

$$
f / G \in A^{2} \text { and }\|f / G\| \leq\|f\| .
$$

Observe that [4 implies that $M=[G]_{z}$. Moreover, since $G$ belongs to $M \ominus z M$,

$$
\int_{\mathbb{D}}\left(|G(z)|^{2}-1\right) z^{k} d \sigma(z)=0, \quad \text { for } k=0,1,2,3, \ldots
$$

In general, any analytic function $G$ which satisfies (3) is said to be $A^{2}$-inner. Such functions are the Bergman space analogs of the classical inner functions, since any classical inner function $g$ has modulus 1 almost everywhere in the unit circle $\mathbb{T}$, and thus satisfies

$$
\int_{\mathbb{T}}\left(|g(z)|^{2}-1\right) z^{k} d m=0, \quad \text { for } k=0,1,2,3, \ldots
$$

Here $d m$ denotes normalized Lebesgue measure in $\mathbb{T}$.

For later reference, we state the result of Aleman, Richter, and Sundberg [4] mentioned previously. 
Lemma 3.3. For every $M$ in $\operatorname{Lat}_{A^{2}}(z)$, we have $M=[M \ominus z M]_{z}$.

\section{WANDERING PROPERTY IN THE Bergman SPACE}

In this section we will prove Theorems 2.1 and 2.2. For this purpose, we will begin with the following straightforward lemma.

Lemma 4.1. Let $\varphi$ be a bounded univalent mapping of the unit disk with $\varphi(0)=0$. If $M \in \operatorname{Lat}_{A^{2}}(z)$, then $M \ominus z M=M \ominus \varphi M$.

Proof. If $M$ is $z$-invariant, it must be $\varphi$-invariant. Thus, it suffices to show that $\varphi M=z M$. The functions $\psi_{1}(z)=\frac{\varphi(z)}{z}$ and $\psi_{2}(z)=\frac{z}{\varphi(z)}$ are in $H^{\infty}$, since the zero of $\varphi$ at the origin is simple and since $\varphi$ is bounded away from zero outside a neighborhood of the origin. Thus $M$ is both $\psi_{1}$-invariant and $\psi_{2}$-invariant. Hence, for any $h$ in $M$, we have $\varphi(z) h(z)=z \psi_{1}(z) h(z) \in z M$, which implies $\varphi M \subseteq z M$, and we have $z h(z)=\varphi(z) \psi_{2}(z) h(z) \in \varphi M$, which implies $z M \subseteq \varphi M$.

We will now prove Theorem 2.1,

Proof of Theorem [2.1. $(\Rightarrow)$ Notice that if $\operatorname{Lat}_{A^{2}}(\varphi)=\operatorname{Lat}_{A^{2}}(z)$, then $[N]_{\varphi}=[N]_{z}$ for every subset $N \subset A^{2}$. Moreover, it follows by Lemma 4.1 that for every $M$ in $\operatorname{Lat}_{A^{2}}(\varphi)$, we have $M \ominus z M=M \ominus \varphi M$. This, the hypothesis, and Lemma 3.3 show that

$$
[M \ominus \varphi M]_{\varphi}=[M \ominus z M]_{\varphi}=[M \ominus z M]_{z}=M,
$$

establishing (i). Furthermore, if $f \in A^{2}$, then $[f]_{\varphi}=[f]_{z}$, and hence (ii) follows by Lemma 4.1

$(\Leftarrow)$ In order to show that $\operatorname{Lat}_{A^{2}}(\varphi)=\operatorname{Lat}_{A^{2}}(z)$, it suffices to prove that $[f]_{z}=$ $[f]_{\varphi}$ for every $f \in A^{2}$. Indeed, if $[f]_{z}=[f]_{\varphi}$ for every $f$, and if $L \in \operatorname{Lat}_{A^{2}}(\varphi)$, then for every $f \in L$, we have $z f \in[f]_{z}=[f]_{\varphi} \subseteq L$, which implies that $L \in \operatorname{Lat}_{A^{2}}(z)$, and hence $\operatorname{Lat}_{A^{2}}(\varphi)=\operatorname{Lat}_{A^{2}}(z)$.

We now verify that $[f]_{z}=[f]_{\varphi}$ for every $f$. To this end, fix $f \in A^{2} \backslash\{0\}$, let $M=[f]_{z}$, and let $G$ be the $A^{2}$-inner function that spans $M \ominus z M$. Since $M$ belongs to $\operatorname{Lat}_{A^{2}}(\varphi)$, it follows by (i), Lemma 4.1 and Lemma 3.3, that

$$
M=[M \ominus \varphi M]_{\varphi}=[M \ominus z M]_{\varphi}=[G]_{\varphi} \subseteq[G]_{z}=M .
$$

In particular, $[G]_{\varphi}=[G]_{z}$. By (ii), we see that $G$ also spans $[f]_{\varphi} \ominus \varphi[f]_{\varphi}$. This, together with (i), shows that

$$
[f]_{\varphi}=\left[[f]_{\varphi} \ominus \varphi[f]_{\varphi}\right]_{\varphi}=[G]_{\varphi}=[G]_{z}=[f]_{z}
$$

as desired.

We remark that if $G$ is any $A^{2}$-inner function, then $G$ spans $M \ominus z M$, where $M=[G]_{z}$. This fact, together with the proof of $(\Leftarrow)$ in Theorem 2.1 implies the following result, which we state as a corollary.

Corollary 4.2. Let $\varphi$ be a bounded univalent mapping of the unit disk for which $\varphi(0)=0$. If $M=[M \ominus \varphi M]_{\varphi}$ for every $M$ in $\operatorname{Lat}_{A^{2}}(\varphi)$, then $[G]_{\varphi}=[G]_{z}$ for every $A^{2}$-inner function $G$.

Observe that in light of Lemma 3.1 one part of Theorem 2.1] says that weak-star generators of $H^{\infty}$ which vanish at the origin have the wandering property in $A^{2}$. It is still an open question whether the converse holds, but Theorem 2.1] does give a partial converse. In addition, one may view Corollary 4.2 as a partial converse. 
Indeed, in order to show that the wandering property of $\varphi$ implies that $\varphi$ is a weakstar generator of $H^{\infty}$, it suffices to show that if $\varphi$ has the wandering property, then $[f]_{\varphi}=[f]_{z}$ for every $f$ in $A^{2}$.

Furthermore, observe that since the constant function $G(z) \equiv 1$ is $A^{2}$-inner, Corollary 4.2 implies that if $\varphi$ is a bounded univalent map with the wandering property in $A^{2}$ for which $\varphi(0)=0$, then $[1]_{\varphi}=[1]_{z}=A^{2}$, and hence polynomials in $\varphi$ are dense in $A^{2}$. We record this formally as a corollary.

Corollary 4.3. Let $\varphi$ be a bounded univalent mapping of the unit disk for which $\varphi(0)=0$. If $\varphi$ has the wandering property in $A^{2}$, then polynomials in $\varphi$ are dense in $A^{2}$.

In order to prove Theorem 2.2, we will make use of a result obtained in 7 . For the sake of completeness, we include the proof. Before stating the result, we introduce some notation. Given $g \in H^{\infty}$ and $f$ in $A^{2}$, let $\Omega=g(\mathbb{D})$, and define the pullback measure $\mu_{f, g}$ on $\Omega$ by

$$
\mu_{f, g}(C)=\int_{g^{-1}(C)}|f|^{2} d \sigma
$$

for every Borel subset $C \subset \Omega$. Let $\mathcal{P}^{2}\left(\Omega, \mu_{f, g}\right)$ denote the closure of analytic polynomials under the norm given by

$$
\|F\|_{\mu_{f, g}}^{2}=\int_{\Omega}|F(w)|^{2} d \mu_{f, g}(w) .
$$

The space $\mathcal{P}^{2}\left(\Omega, \mu_{f, g}\right)$ is a Hilbert space, equipped with the inner product

$$
\langle F, G\rangle_{\mu_{f, g}}=\int_{\Omega} F(w) \overline{G(w)} d \mu_{f, g}(w),
$$

and it has a reproducing kernel $K_{\mu_{f, g}}(z, w)$, which is analytic in $z$, and antianalytic in $w$, for $z, w \in \Omega$. (See [7] for details.)

The next result will be used in the proof of Theorem 2.2 .

Proposition 4.4. Let $g \in H^{\infty}$, and let $M=[f]_{g}$ for some $f$ in $A^{2}$. Then $M \ominus g M$ is one-dimensional and is spanned by the function $f(z) K_{\mu_{f, g}}(g(z), 0)$.

Proof. Let $\Omega=g(\mathbb{D})$, and suppose that $\theta$ belongs to $M \ominus g M$. Then there is a sequence $\left\{P_{n}\right\}$ of polynomials for which $\left(P_{n} \circ g\right) f$ converges to $\theta$ in $A^{2}$-norm. Therefore, the sequence $\left\{P_{n}\right\}$ must converge in $\mathcal{P}^{2}\left(\Omega, \mu_{f, g}\right)$-norm to some function $\Theta \in \mathcal{P}^{2}\left(\Omega, \mu_{f, g}\right)$. In particular, $\theta=(\Theta \circ g) f$. Now let $\Psi$ be any function in $\mathcal{P}^{2}\left(\Omega, \mu_{f, g}\right)$, and let $\psi=(\Psi \circ g) f$. It is clear that $\psi \in M$. Therefore,

$$
\int_{\Omega} \Theta(z) \overline{z \Psi(z)} d \mu_{f, g}(z)=\int_{\mathbb{D}} \theta \overline{g \psi} d \sigma=0
$$

since $\theta \in M \ominus g M$. Since $z \Psi(z)$ represents an arbitrary function in $\mathcal{P}^{2}\left(\Omega, \mu_{f, g}\right)$ vanishing at the origin, it follows that $\Theta(z)=\lambda K_{\mu_{f, g}}(z, 0)$ for some complex number $\lambda$. Hence $\theta(z)=\lambda f(z) K_{\mu_{f, g}}(g(z), 0)$, as desired.

We remark that Proposition 4.4 can be used to reformulate condition (ii) of Theorem [2.1] Indeed, condition (ii) of Theorem 2.1 may be replaced with the condition

(ii') for every $f$ in $A^{2}$, we have $K_{\mu_{f, \varphi}}(\varphi(z), 0)=\lambda K_{\mu_{f, z}}(z, 0)$ for some $\lambda$ in $\mathbb{C}$. 
It is not clear, however, whether this condition is easily verified for functions other than the weak-star generators of $H^{\infty}$.

We now turn to the proof of Theorem [2.2.

Proof of Theorem 2.2, In order to prove that (A) implies (B), suppose that $g$ is univalent and let $M=[f]_{z}$ for some $f$ in $A^{2}$. Then $M=[G]_{z}$ where $G$ is the extremal function for $M$. Since $G$ is $A^{2}$-inner, it follows that $M=[G]_{g}$ by Corollary 4.2 Hence (A) implies (B). That (B) implies (C) is obvious.

We will now prove that (C) implies (A). Suppose that $M=[f]_{z}=[\tilde{f}]_{g}$ for some $f, \tilde{f}$ in $A^{2}$, and let $G$ be the extremal function for $M$. Since $g(0)=0$, it is clear that $M \ominus z M \subseteq M \ominus g M$. However, Proposition 4.4 implies that $M \ominus g M$ is one-dimensional, and thus $G$ spans both $M \ominus z M$ and $M \ominus g M$. The wandering property of $g$ now implies that $[G]_{g}=M=[G]_{z}$. If $P$ is any polynomial, then $P G \in M$, and hence there is a sequence $\left\{P_{n}\right\}$ of polynomials for which $\left(P_{n} \circ g\right) G$ converges to $P G$ in $A^{2}$-norm. Since $G$ has the property (2), it follows that $\left(P_{n} \circ g\right)$ converges to $P$ in $A^{2}$-norm. Hence, polynomials in $g$ are dense in $A^{2}$, and so $g$ must be univalent.

\section{WANDERING PROPERTY IN THE HARDY SPACE}

The results in the previous section have Hardy space relatives. Specifically, in the proofs of Lemma 4.1 and Theorem 2.1, we may replace $A^{2}$ with $H^{2}$, and replace " $A^{2}$-inner" with "classical inner," and easily obtain the following Hardy space version of Theorem 2.1.

Theorem 5.1. Let $\varphi$ be a bounded univalent mapping of $\mathbb{D}$, with $\varphi(0)=0$. Then $\operatorname{Lat}_{H^{2}}(\varphi)=\operatorname{Lat}_{H^{2}}(z)$ if and only if

(i) $M=[M \ominus \varphi M]_{\varphi}$ for every $M$ in $\operatorname{Lat}_{H^{2}}(\varphi)$, and

(ii) $[f]_{\varphi} \ominus \varphi[f]_{\varphi}=[f]_{z} \ominus z[f]_{z}$ for every $f$ in $H^{2}$.

Similarly, we easily obtain the following Hardy space version of Corollary 4.3 .

Corollary 5.2. Let $\varphi$ be a bounded univalent mapping of the unit disk for which $\varphi(0)=0$. If $\varphi$ has the wandering property in $H^{2}$, then polynomials in $\varphi$ are dense in $H^{2}$.

\section{Concluding Remarks}

As mentioned, weak-star generators of $H^{\infty}$ (which vanish at the origin) have the wandering property in both $A^{2}$ and $H^{2}$. In both settings, it would be interesting to know whether the weak-star generators of $H^{\infty}$ are the only bounded univalent mappings with the wandering property. If condition (i) implies condition (ii) of Theorem 2.1, then the weak-star generators are the only bounded univalent mappings with the wandering property in $A^{2}$. A similar remark may be made for the Hardy space regarding Theorem 5.1. This and Proposition 4.4 point to a need to investigate the kernels $K_{\mu_{f, \varphi}}$.

We conclude the paper by verifying Corollary 2.3 that is, we will now show that in both the Bergman and Hardy space settings, there do in fact exist bounded univalent mappings which fail to have the wandering property. We will address the $H^{2}$ case first. 
Examples in the Hardy space setting. To give an example of a bounded univalent mapping which does not have the wandering property in the Hardy space $H^{2}$, we will simply appeal to Corollary 6.1 below, whose proof follows from Corollary 5.2 and from an argument given in [6].

Corollary 6.1. Let $\varphi$ be a bounded univalent mapping of the unit disk for which $\varphi(0)=0$. If $\varphi$ has the wandering property in $H^{2}$, then $\varphi$ is univalent a.e. on $\mathbb{T}$.

Proof. Suppose that $\varphi$ has the wandering property in $H^{2}$. Then by Corollary 5.2 polynomials in $\varphi$ are dense in $H^{2}$. Therefore, there is a sequence $\left\{P_{n}\right\}$ of polynomials for which $P_{n} \circ \varphi$ converges in $H^{2}$-norm to the identity map. Thus, there is a subset $E \subset \mathbb{T}$ of full measure and a subsequence $\left\{P_{n_{j}}\right\}$ for which $P_{n_{j}}(\varphi(w)) \rightarrow w$ for each $w$ in $E$. (See, for example, Corollary 2.32 of [9].) In particular, it follows that if $w_{1}$ and $w_{2}$ are in $E$ with $\varphi\left(w_{1}\right)=\varphi\left(w_{2}\right)$, then $w_{1}=w_{2}$.

It follows from Corollary 6.1 that any univalent map $\varphi$ (vanishing at the origin) which takes the unit disk onto a disk minus finitely many radial slits cannot have the wandering property in $H^{2}$, since such a mapping cannot be univalent a.e. on $\mathbb{T}$. (We caution the reader that we cannot conclude here that every map $\varphi$ which takes $\mathbb{D}$ onto a slitted disk fails to have the wandering property in $H^{2}$. Indeed, Akeroyd [1] has constructed a Jordan curve $\Gamma$ which connects 0 to 1 and lies in $\mathbb{D}$, for which the mapping $\varphi$ from $\mathbb{D}$ onto $\mathbb{D} \backslash \Gamma$ has the remarkable property that polynomials in $\varphi$ are dense in $H^{2}$, and consequently $\varphi$ is univalent a.e. on $\mathbb{T}$. So for this example, Corollaries 5.2 and 6.1 do not apply. It is not known whether this map $\varphi$ has the wandering property in $H^{2}$.)

Furthermore, there is a large class of crescents $\Omega$ for which the map $\varphi$ taking $\mathbb{D}$ onto $\Omega$ cannot have the wandering property in $H^{2}$. Indeed, in [2] and [3] there is exhibited a collection of crescents $\Omega$ for which the map $\varphi$ from $\mathbb{D}$ onto $\Omega$ has the property that polynomials in $\varphi$ are not dense in $H^{2}$, and so by Corollary 5.2 any such $\varphi$ cannot have the wandering property in $H^{2}$. (However, we remark that even if $\Omega$ is a crescent bounded by two internally tangent circles, and if $\varphi$ maps $\mathbb{D}$ onto $\Omega$, then as shown in [2], polynomials in $\varphi$ are dense in $H^{2}$, and it is still an open problem to determine whether this map has the wandering property in $H^{2}$.)

Examples in the Bergman space setting. Using the work of [3], we can also show that there are bounded univalent mappings without the wandering property in the Bergman space. Specifically, given a bounded domain $\Omega$, let $A^{2}(\Omega)$ denote the Bergman space on $\Omega$, that is, the Hilbert space of functions $f$ analytic in $\Omega$ for which the norm

$$
\left(\int_{\Omega}|f|^{2} d A\right)^{1 / 2}
$$

is finite. Here $d A$ denotes Lebesgue area measure in $\Omega$. A function $f$ in $A^{2}(\Omega)$ is said to be cyclic in $A^{2}(\Omega)$ provided $\{P f: P$ is a polynomial $\}$ is dense in $A^{2}(\Omega)$.

Suppose that $\varphi$ is a bounded univalent mapping which has the wandering property in $A^{2}$, and that $\varphi(0)=0$. If $\Omega=\varphi(\mathbb{D})$, then since polynomials in $\varphi$ are dense in $A^{2}$ (by Corollary 4.3), it follows that the function $\left(\varphi^{-1}\right)^{\prime}$ is cyclic in $A^{2}(\Omega)$, by a simple change of variables argument. We record this formally as a corollary.

Corollary 6.2. Let $\varphi$ be a bounded univalent mapping of the unit disk for which $\varphi(0)=0$, and let $\Omega=\varphi(\mathbb{D})$. If $\varphi$ has the wandering property in $A^{2}$, then $\left(\varphi^{-1}\right)^{\prime}$ is cyclic in $A^{2}(\Omega)$. 
However, in [3] it was shown that if $\Omega$ is a disk minus finitely many radial slits, then $A^{2}(\Omega)$ has no cyclic elements. Consequently, any mapping $\varphi$, with $\varphi(0)=0$, which takes $\mathbb{D}$ onto such a domain $\Omega$, cannot have the wandering property in $A^{2}$. Furthermore, [3] also gave a collection of crescents $\Omega$ for which $A^{2}(\Omega)$ has no cyclic elements, and so any mapping $\varphi$, vanishing at the origin and taking $\mathbb{D}$ onto such a crescent $\Omega$, cannot have the wandering property in $A^{2}$.

\section{ACKNOWLEDGMENT}

The author would like to thank John Akeroyd, Peter Duren, and Michael Stessin for many helpful discussions about the topics addressed in this paper. In addition, the author would like to thank the referee for providing many helpful suggestions for the improvement the paper, including a simplification of the statement and the proof of Theorem 2.1

\section{REFERENCES}

[1] J. Akeroyd, "Density of the polynomials in the Hardy space of certain slit domains," Proc. Amer. Math. Soc. 115 (1992), 1013-1021. MR 92j:30030

[2] _ "Polynomial approximation in the mean with respect to harmonic measure on crescents II," Michigan Math. J. 39 (1992), 35-40. MR 93g:30052

[3] J. Akeroyd, D. Khavinson, H. Shapiro, "Remarks concerning cyclic vectors in Hardy and Bergman spaces," Michigan Math. J. 38 (1991), 191-205. MR 92h:47034

[4] A. Aleman, S. Richter, and C. Sundberg, "Beurling's theorem for the Bergman space," Acta Math. 177 (1996), 275-310. MR 98a:46034

[5] A. Beurling, "On two problems concerning linear transformations in Hilbert space," Acta Math. 81 (1948), 239-255. MR 10:381e

[6] P. Bourdon, "Density of the polynomials in Bergman spaces," Pacific J. Math. 130, no. 2 (1987), 215-221. MR 89a:46060

[7] B. Carswell, P. Duren, and M. Stessin, "Multiplication invariant subspaces of the Bergman space," Indiana Univ. Math. J., to appear.

[8] P. Duren and A. Schuster, Bergman Spaces, American Mathematical Society, Providence, R.I., to appear.

[9] G. B. Folland, Real Analysis: Modern Techniques and Their Applications, Wiley, New York, 1984. MR 86k:28001

[10] H. Hedenmalm, "A factorization theorem for square area-integrable analytic functions", J. Reine Angew. Math. 422 (1991), 45-68. MR 93c:30053

[11] H. Hedenmalm, B. Korenblum, and K. Zhu, Theory of Bergman Spaces, Springer, New York, 2000. MR 2001c: 46043

[12] D. Khavinson, T. L. Lance, and M. I. Stessin, "Wandering property in the Hardy space," Michigan Math. J. 44 (1997), 597-606. MR 99a:47012

[13] D. Sarason, "Invariant subspaces and unstarred operator algebras," Pacific J. Math. 17 (1966), 145-162. MR 33:590

[14] — "Weak-star generators of $H^{\infty}$," Pacific J. Math. 17, no. 3 (1966), 519-528. MR 35:2151

Department of Mathematics, University of Michigan, Ann Arbor, Michigan 48109

E-mail address: carswell@umich.edu

URL: http://www.math.lsa.umich.edu/ ${ }^{\text {carswell }}$ 